\title{
Gravitational depolarization of ultracold neutrons: Comparison with data
}

S. Afach, ${ }^{1,2,3}$ N. J. Ayres, ${ }^{4}$ C. A. Baker, ${ }^{5}$ G. Ban, ${ }^{6}$ G. Bison, ${ }^{1}$ K. Bodek, ${ }^{7}$ M. Fertl, ${ }^{1,2, \dagger}$ B. Franke, ${ }^{1,2, *}$ P. Geltenbort,${ }^{8}$ K. Green, ${ }^{5}$ W. C. Griffith, ${ }^{4}$ M. van der Grinten, ${ }^{5}$ Z. D. Grujic,${ }^{9}$ P. G. Harris ${ }^{4,8}$ W. Heil, ${ }^{10}$ V. Hélaine, ${ }^{6, t}$ P. Iaydjiev, ${ }^{5, \|}$ S. N. Ivanov, ${ }^{5,9}$ M. Kasprzak, ${ }^{9, *}$ Y. Kermaidic, ${ }^{11}$ K. Kirch, ${ }^{1,2}$ H.-C. Koch, ${ }^{9,10}$ S. Komposch, ${ }^{1,2}$ A. Kozela, ${ }^{12}$ J. Krempel, ${ }^{2}$ B. Lauss, ${ }^{1}$ T. Lefort, ${ }^{6}$ Y. Lemière, ${ }^{6}$ M. Musgrave ${ }^{4}$ O. Naviliat-Cuncic, ${ }^{6, \dagger}$ J. M. Pendlebury, ${ }^{4,{ }^{*}}$ F. M. Piegsa, ${ }^{2}$ G. Pignol, ${ }^{11}$ C. Plonka-Spehr, ${ }^{13}$ P. N. Prashanth, ${ }^{14}$ G. Quéméner, ${ }^{6}$ M. Rawlik, ${ }^{2}$ D. Rebreyend, ${ }^{11}$ D. Ries, ${ }^{1,2}$ S. Roccia, ${ }_{1}{ }^{4}$ D. Rozpedzik, ${ }^{7}$ P. Schmidt-Wellenburg, ${ }^{1}$ N. Severijns ${ }^{14}$ D. Shiers, ${ }^{4}$ J. A. Thorne, ${ }^{4}$ A. Weis, ${ }^{9}$ E. Wursten, ${ }^{14}$ J. Zejma, J. Zenner, ${ }^{1,2,13}$ and G. Zsigmond ${ }^{1}$

${ }^{1}$ Paul Scherrer Institute, CH-5232 Villigen PSI, Switzerland

${ }^{2}$ ETH Zürich, Institute for Particle Physics, CH-8093 Zürich, Switzerland

${ }^{3}$ Hans Berger Department of Neurology, Jena University Hospital, D-07747 Jena, Germany

${ }^{4}$ Department of Physics and Astronomy, University of Sussex, Falmer, Brighton BN1 9QH, United Kingdom

${ }^{5}$ STFC Rutherford Appleton Laboratory, Harwell, Didcot, Oxon OX11 OQX, United Kingdom

${ }^{6}$ LPC Caen, ENSICAEN, Université de Caen, CNRS/IN2P3, Caen, France

${ }^{7}$ Marian Smoluchowski Institute of Physics, Jagiellonian University, 30-348 Cracow, Poland

${ }^{8}$ Institut Laue-Langevin, CS 20156, F-38042 Grenoble Cedex 9, France

${ }^{9}$ Physics Department, University of Fribourg, CH-1700 Fribourg, Switzerland

${ }^{10}$ Institut für Physik, Johannes-Gutenberg-Universität, D-55128 Mainz, Germany

${ }^{11}$ LPSC, Université Grenoble Alpes, CNRS/IN2P3, Grenoble, France

${ }^{12}$ Henryk Niedwodniczański Institute for Nuclear Physics, Cracow, Poland

${ }^{13}$ Institut für Kernchemie, Johannes-Gutenberg-Universität, Mainz, Germany

${ }^{14}$ Instituut voor Kern- en Stralingsfysica, Katholieke Universiteit Leuven, B-3001 Leuven, Belgium

${ }^{15}$ CSNSM, Université Paris Sud, CNRS/IN2P3, Orsay, France

(Received 22 June 2015; published 22 September 2015)

We compare the expected effects of so-called gravitationally enhanced depolarization of ultracold neutrons to measurements carried out in a spin-precession chamber exposed to a variety of vertical magnetic-field gradients. In particular, we have investigated the dependence upon these field gradients of spin-depolarization rates and also of shifts in the measured neutron Larmor precession frequency. We find excellent qualitative agreement, with gravitationally enhanced depolarization accounting for several previously unexplained features in the data.

\section{INTRODUCTION}

Ultracold neutrons (UCN) are neutrons of extremely low energy, typically $\sim 200 \mathrm{neV}$ or less, which can be stored in material bottles and which are routinely used in experiments

\footnotetext{
* Deceased.

†resent address: University of Washington, Seattle, Washington, USA.

*Present address: Max-Planck-Institute of Quantum Optics, Garching, Germany.

${ }^{\S}$ Corresponding author.

p.g.harris@sussex.ac.uk

${ }^{1}$ On leave of absence from Institute of Nuclear Research and Nuclear Energy, Sofia, Bulgaria.

"On leave of absence from Petersburg Nuclear Physics Institute, Russia.

** Present address: Instituut voor Kern- en Stralingsfysica, Katholieke Universiteit Leuven, B-3001 Leuven, Belgium.

${ }^{\dagger}$ Present address: Michigan State University, East Lansing, Michigan, USA.

\#Present address: LPSC, Université Grenoble Alpes, CNRS/ IN2P3, Grenoble, France.
}

such as the ongoing search for the neutron electric dipole moment (nEDM). Collisions with the containing walls are elastic, so the UCN never thermalize. Being of such low energy, they "sag" under gravity, and rather than being distributed uniformly throughout their storage vessel their density decreases with increasing height, with each specific energy group having its own center of mass. In the presence of a vertical magnetic-field gradient, the average magnetic field sampled by the neutrons will therefore depend upon the neutron energy. The implications of this stratification have been discussed in earlier work [1-3], but, in summary, it results in a relative dephasing of the neutrons in different energy bins, which then alters the measured Larmor spinprecession frequency. This phenomenon is referred to as gravitationally enhanced depolarization, in contrast to the intrinsic depolarization that takes place within each energy bin as a result of the neutrons sampling different fields as they move around the storage volume. A key distinction is the asymmetric nature of the gravitationally induced dephasing, as shown in Fig. 3 of [1], with the lowest-energy 
neutrons playing a particularly crucial role. The resulting nonlinearities in frequency response as a function of applied magnetic-field gradients represent potential sources of systematic uncertainty in precision experiments such as nEDM searches [4-7]. Since such experiments provide tight constraints on physics beyond the Standard Model, with consequent implications for particle theory and cosmology, a full understanding of the phenomenon is essential.

In this paper, we compare our experimentally measured results, both in terms of frequency shifts and of depolarization rates, with those anticipated from theoretical calculations. We begin in Sec. II with a discussion of the spectrum of UCN within our storage cell; this underlies the subsequent calculations of the gravitationally enhanced depolarization. We give an overview of the calculations themselves in Sec. III. In Sec. IV we discuss the basic intrinsic-depolarization mechanisms, which are revealed to make only a minor contribution to the frequency shifts. We then present, in Sec. V, a direct comparison of the anticipated and measured polarization $\alpha$ remaining after $180 \mathrm{~s}$ of storage in a range of applied $B$-field gradients. In Secs. VI and VII, we consider the frequency shifts that arise from this phenomenon, before finally discussing in Sec. VIII the possible implications for nEDM experiments, including the current world limit in particular.

The measurements described in this paper form part of a program of work [7] aimed at an accurate determination of the nEDM, currently being carried out at the new highintensity UCN source [8] based at the Paul Scherrer Institute (PSI). The experimental apparatus and procedures are described in substantial detail in [9]. The apparatus is based upon that used [10] in an earlier nEDM measurement at the Institut Laue-Langevin (ILL) [5], but substantially upgraded with the incorporation, in particular, of an array of Cs magnetometers [11], a system for the simultaneous detection of both neutron spin states [12], and a set of active compensation coils that provide dynamic shielding of external magnetic fields [13].

The $1 \mu \mathrm{T}$ magnetic holding field $B_{0}$ within the EDM spectrometer is primarily vertical, so $B_{0} \approx B_{z}$, although there are small transverse components $B_{x}, B_{y}$ present at the $\sim$ few nT level. We define

$$
B_{0}=B_{z}+\frac{1}{2} \frac{B_{t}^{2}}{B_{z}}
$$

where $B_{t}^{2}=B_{x}^{2}+B_{y}^{2}$.

In order to compensate for changes in $B_{0}$, a cohabiting atomic-mercury magnetometer [14] is used to make precise real-time measurements of the volume-averaged field within the UCN storage cell. Under an applied vertical magnetic-field gradient $\partial B_{z} / \partial z$, the measured ratio $R$ of neutron to mercury precession frequencies undergoes a relative change of, to first order,

$$
\frac{\delta R}{R}=\frac{1}{B_{0}} \frac{\partial B_{0}}{\partial z} \Delta h,
$$

where $\Delta h$ is the difference between the centers of mass of the populations of (thermal) mercury atoms and (ultracold) neutrons. Precise measurements of this frequency-ratio dependence are the subject of [9]. As we shall see, gravitationally enhanced depolarization can impose a substantial nonlinearity in this relationship: indeed, we are unaware of any other mechanism that can do so to the extent required to match our observations.

\section{INPUT SPECTRA}

The extent of gravitational depolarization clearly depends heavily upon the spectrum of stored UCN. We have recently carried out a series of measurements using a spin-echo technique [15], from which we were able to derive the distribution of energies of UCN remaining after $220 \mathrm{~s}$ of storage in our apparatus. The resulting fitted spectrum is parametrized by

$$
p(E)=A \cdot E^{1 / 2} \cdot \frac{1}{1+e^{\frac{E_{0}-E}{\Delta E_{0}}}} \cdot \frac{1}{1+e^{\frac{E-E_{1}}{\Delta E_{1}}}},
$$

where $A$ is an arbitrary normalization, $E_{0}=7.7 \mathrm{neV}$, $\Delta E_{0}=1 \mathrm{neV}, \quad E_{1}=28.7 \mathrm{neV}$, and $\Delta E_{1}=6.25 \mathrm{neV}$. The form of this parametrization is based on a very general distribution $n(E) \mathrm{d} E \propto E^{1 / 2} \mathrm{~d} E$ from the low-energy tail of a Maxwell-Boltzmann distribution, allowing for low- and high-energy cutoffs.

The spin-echo technique is particularly sensitive to the presence of low-energy UCN, but once the neutrons start to populate the bottle more or less uniformly it becomes increasingly difficult to distinguish between different energies. This is clear from Figs. 2(a) and 2(b) in [15], where the low-energy tails are fitted well but the high-energy region produces less reliable results. Furthermore, the spinecho measurements were carried out at a storage time of $220 \mathrm{~s}$, whereas the polarization and frequency-ratio measurements used in the current analysis were carried out at a storage time of $180 \mathrm{~s}$. On both counts, therefore, we should not be surprised if the actual spectrum were to be somewhat firmer than that arising from the spin-echo measurement.

We have also used the package MCUCN [16] to carry out a detailed simulation of the UCN within our apparatus, which yields an alternative estimate of the spectrum after $180 \mathrm{~s}$ of storage. The simulation is based upon very detailed modeling of the PSI UCN source, beam line, and guides, as well as of the nEDM storage vessel. The latter consists of aluminum electrodes coated with diamond-like carbon, which form the floor and roof, and between them an insulating cylindrical polystyrene ring coated with deuterated polystyrene to provide radial containment. 
The simulation accounts for losses during storage both from $\beta$ decay and as a result of wall collisions [17], with the "loss-per-bounce" factor $f=W / V$ (where $W, V$ are the imaginary and real parts, respectively, of the Fermi potential) set to a common value of $3 \times 10^{-4}$ for the electrodes and for the insulator walls. In fact, although $V$ is well known, $W$ is difficult to determine. Losses are likely to be dominated by hydrogen that has diffused into the containing surfaces, and can-because it has an extremely high incoherent-scattering cross section-substantially influence loss rates without significantly altering the surface potential $V$. Using an average value of $f$ for all of the containing walls appears a reasonable approach, and the number here arises from earlier simulation-based studies [18] that were tuned to match the observed numbers of neutrons stored as a function of time.

Any damage to the coatings on either the electrodes or the insulating walls would result in an area of reduced Fermi potential that would preferentially deplete the higher-energy neutrons. The same is true of small gaps, which may not be completely accurately modeled in the simulation. The actual spectrum, therefore, is likely to be somewhat softer than the simulation would suggest.

The spectra resulting from the Monte Carlo (MC) simulation and spin-echo (SE) studies are shown in Fig. 1. It is useful to refer to UCN energies $E$ in terms of the maximum height $\epsilon=E /(m g)$ attainable under gravity in a trap with no vertical confinement, and to this end the abscissa is in units of $\mathrm{cm}$.

We note here for clarity that the energies are defined to be the kinetic energies that the UCN would have at the floor of the storage vessel, i.e. at the bottom electrode.

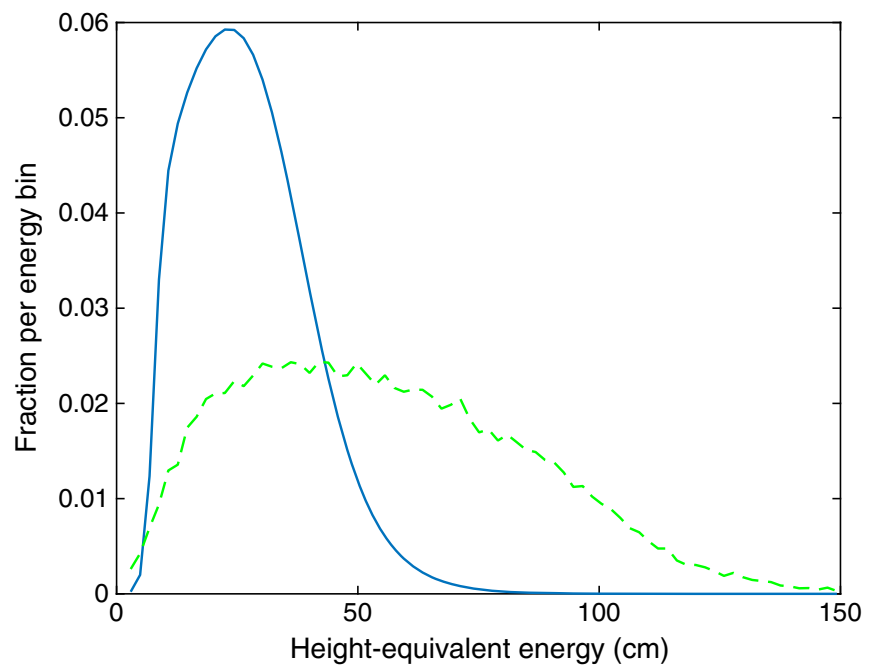

FIG. 1 (color online). Estimated energy spectrum of stored UCN from spin-echo measurement (blue solid line) and from simulation (green dashed line). Each is normalized to give unit total area under the curve.

\section{CAlCulations}

Calculations of the gravitationally enhanced depolarization effect are relatively straightforward to carry out. Phasespace arguments [19] show that the variation of density $\rho$ with height $z$ of UCN of height-equivalent energy $\epsilon$ is (for $z<\epsilon$ ) given by

$$
\rho(z, \epsilon)=\rho(0, \epsilon)\left(1-\frac{z}{\epsilon}\right)^{1 / 2},
$$

assuming sufficiently diffuse reflections for the phase space to be approximately uniformly populated on a time scale short compared to the storage time. (Our Monte Carlo simulations confirm that this typically takes place within $5 \mathrm{~s}$ of closing the UCN shutter if more than $10 \%$ of reflections are diffuse, and more quickly still with higher diffusivity and also for the more energetic of the neutrons in our spectrum.) The height distribution of Eq. (4) will then be reflected in the distribution of average magnetic fields to which UCN of any particular energy will be exposed, from which the distribution (appropriate to that UCN energy) of integrated phases acquired after $180 \mathrm{~s}$ of free Larmor precession can be calculated. This procedure is carried out for all UCN energies across the spectrum, accounting for the relative populations of each energy bin. The resulting total array of integrated phases $\phi_{i}$ is then subject to a Ramsey-type analysis, where, as discussed in [1], the net frequency is determined by the reference phase

$$
\hat{\phi}=2 n \pi+\tan ^{-1}\left(\frac{\left\langle\sin \phi_{i}\right\rangle}{\left\langle\cos \phi_{i}\right\rangle}\right)
$$

divided by the Ramsey coherence time (180 s in this case). Note that the $2 n \pi$ term, which arises because the Ramsey technique measures phases modulo $2 \pi$, is relatively easily accounted for by, for example, monitoring the discrepancy between the reference phase and the mean of the array of time-integrated phases, and adding (or subtracting) multiples of $2 \pi$ as appropriate to compensate. The nonlinearities in response referred to earlier primarily arise when the lowest-energy UCN, which do not reach the roof of the storage trap, have an integrated phase that differs by more than $\pi$ radians from the reference phase: they then "wrap around" and appear to enhance the high-energy tail of the distribution. We will refer to this phenomenon as "Ramsey wrapping."

Effects due to intrinsic depolarization, arising from both vertical and horizontal field gradients, can also be included by appropriate weighting of the distribution of phases. We discuss this in some detail in the following section.

\section{INTRINSIC DEPOLARIZATION MECHANISMS}

Detailed calculations of intrinsic depolarization within magnetic-field gradients have been carried out 
elsewhere [20-27]. There are four relevant scenarios to consider: vertical gradients $\partial B_{z} / \partial z$; horizontal gradients of the form $\partial B_{z} / \partial x$; transverse fields $B_{t}$ and their gradients; and wall collisions involving small magnetic impurities. We present here some simple and rather intuitive models of the depolarization mechanisms, and we discuss possible implications for the polarization and frequency-ratio measurements. Throughout this section, where calculations are dependent upon an input spectrum we use that derived from the SE measurement.

\section{A. Vertical gradients: $\partial B_{z} / \partial z$}

Here we consider UCN confined within a vertical magnetic-field gradient $\partial B_{z} / \partial z$. Let the confining trap be a cylinder of height $H$ and radius $r$. Following [19], we replace $H$ with an "effective height" $\mathcal{H}(\epsilon)$ which is simply defined as the lesser of $H, \epsilon$; this accommodates UCN with energies too low to reach the roof of the trap.

The following method is based upon that outlined in the derivation of Eq. (68) in [28]. We shall consider our trap to be divided by a horizontal plane into two halves, with average field strengths that each differ from the field at the center plane by $\Delta B_{z}=\left(\partial B_{z} / \partial z\right) \mathcal{H} / 4$. Let the average dwell time for UCN in each half of the trap be $t_{w}$. Using the standard kinetic-theory result (due to Clausius [29]) that the rate of wall collisions per particle is $A v /(4 V)$, where $A$ is here the area of the dividing plane, $V$ is half of the containing volume and $v$ is the speed of the particles, we can calculate the rate of passage between the two halves. From this we find

$$
t_{w}=\frac{2 \mathcal{H}}{v} .
$$

Consider now a single UCN. Effectively, a coin is tossed once every $t_{w}$ to determine which side of the trap the neutron is in. Over a storage time $t$, this decision is therefore made $N=t / t_{w}$ times. The number of times $n$ for which the UCN is on the side with the stronger field is binomially distributed with mean $N / 2$ and variance $N / 4$. The additional $\int B \cdot d t$ experienced by this $\mathrm{UCN}$ is $\sim 2(n-N / 2) t_{w} \Delta B_{z}$ (where the factor 2 accounts for the fact that when it is not in the stronger-field region it is in the weaker-field region). Multiplying this by the neutron gyromagnetic ratio $\gamma_{n}$ gives the extra precession angle $\theta_{t}$ away from the mean. The polarization is the average projection upon the mean precession vector, and using

$$
e^{-t / T_{2}} \sim 1-\frac{t}{T_{2}}+\cdots
$$

where $T_{2}$ is the transverse spin-relaxation time, together with $\cos \theta \sim 1-\theta^{2} / 2+\cdots$, we find that

$$
\frac{t}{T_{2, \mathrm{vgi}}} \sim \frac{\left\langle\theta_{t}^{2}\right\rangle}{2}=2\left(\frac{N}{4}\right) \gamma_{n}^{2} \Delta B_{z}^{2} t_{w}^{2}
$$

where the subscript "vgi" stands for "vertical gradient, intrinsic." This yields

$$
T_{2, \mathrm{vgi}} \sim \frac{2}{\gamma_{n}^{2} \Delta B_{z}^{2} t_{w}}=\frac{16 v}{\mathcal{H}^{3} \gamma_{n}^{2}\left(\partial B_{z} / \partial z\right)^{2}}
$$

The upper solid blue line in Fig. 2 shows the prediction of Eq. (9), and despite the rather crude nature of its derivation it is seen to lie nicely between the results of our simulations for completely diffuse reflections (green circles) and for the case where the probability of specular reflections is $80 \%$ (green squares). We therefore use Eq. (9) in our calculations going forward, bearing in mind nonetheless that there is some uncertainty in the size of its contribution. The simulated results also appear in Fig. 2 of [1], where it is shown that the expected dependence upon $\left(\partial B_{z} / \partial z\right)^{2}$ holds true over a wide range of gradients.

After a measurement time $t$, the polarization $\alpha$ is reduced by a factor

$$
e^{-t / T_{2, \text { vgi }}} \sim\left(1-\frac{t \mathcal{H}^{3} \gamma_{n}^{2}}{16 v}\left(\frac{\partial B_{z}}{\partial z}\right)^{2}+\cdots\right)
$$

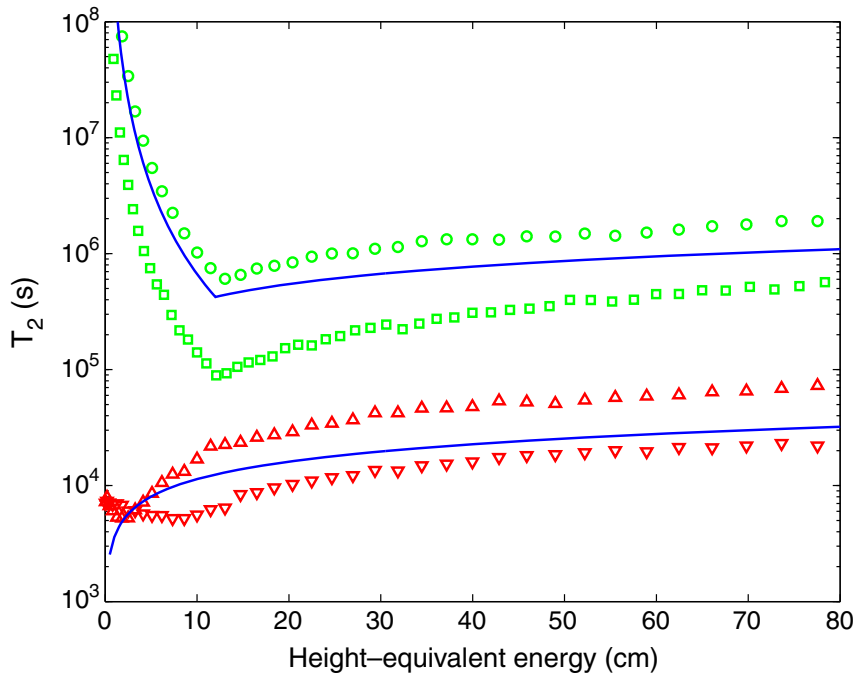

FIG. 2 (color online). Intrinsic depolarization times $T_{2}$ for UCN in $10 \mathrm{pT} / \mathrm{cm}$ magnetic-field gradients. The upper set is for vertical gradients $\partial B_{z} / \partial z$ with the results of simulations for completely diffuse (green circles) and 80\% specular (green squares) reflections; the solid blue line is the analytical approximation of Eq. (9). The lower set is for transverse gradients in the vertical field, of the form $\partial B_{z} / \partial x$. Red upwards-pointing (downwards-pointing) triangles are for diffuse (80\% specular) reflections, and the solid blue line represents the analytical approximation of Eq. (14). $T_{2}$ scales as the inverse square of the applied gradient. 
which implies a parabolic profile to the dependence of $\alpha$ upon the vertical magnetic-field gradient. We see this in Fig. 3, which shows (dotted black line) the spectrumweighted average $\alpha$ as a function of the applied vertical gradient.

We can now proceed to make a rough estimate of the extent to which this intrinsic depolarization may result in a shift in the measured neutron frequency. Intuitively, for example, it might seem that if low-energy UCN depolarize more quickly than their high-energy counterparts, they would have less of a role to play in determining the frequency (since the uncertainty on the frequency measurement is inversely proportional to the polarization $\alpha$ ). In consequence, the frequency measurement may appear to arise from a somewhat stiffer spectrum than is actually the case, thereby raising the effective center of mass of the neutrons and reducing the factor $\Delta h$ in Eq. (2). In order to consider this, let us for the time being imagine that we can make absolute frequency measurements without the complication of the modulo $2 \pi$ arising from the Ramsey measurement, thus ignoring the Ramsey wrapping that is characteristic of the behavior of the lowest-energy UCN [1].

By definition, the height difference $\Delta h$ between the centers of mass of mercury and UCN [Eq. (2)] is

$$
\Delta h=\hat{z}_{\mathrm{Hg}}-\frac{\iint z \rho(z, \epsilon) d z d \epsilon}{\iint \rho(z, \epsilon) d z d \epsilon}
$$

where $\hat{z}_{\mathrm{Hg}}$ is the center of mass of the mercury atoms. We note in passing the standard result that, for $\epsilon \gg H$, $\Delta h=H^{2} /(24 \epsilon)$. When $\epsilon<H, \Delta h=H / 2-0.4 \epsilon$ instead;

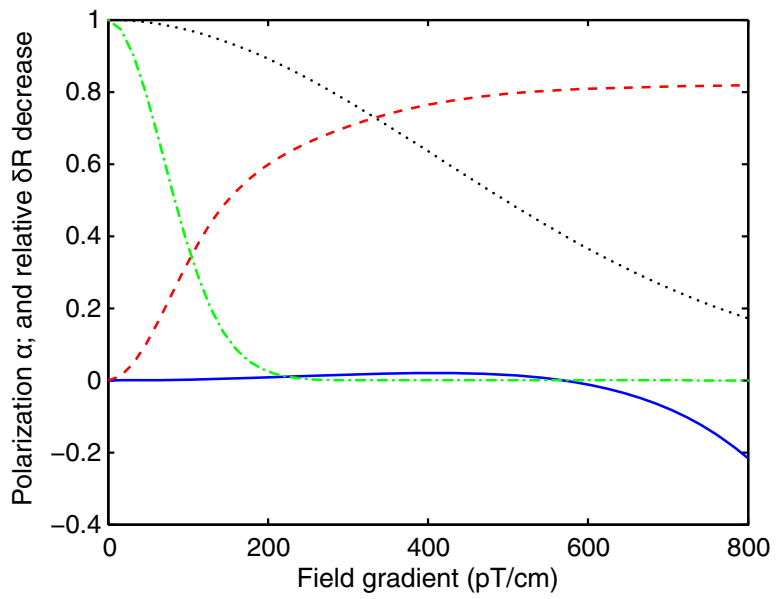

FIG. 3 (color online). Polarization $\alpha$, and fractional decrease in the shift $\delta R$ in frequency ratio, arising from intrinsic depolarization only, after $180 \mathrm{~s}$ of storage. Black dotted (green dot dashed): $\alpha$ from $\partial B_{z} / \partial z\left(\partial B_{z} / \partial x\right)$. Blue solid (red dashed): relative decrease in $\delta R$, i.e. in the slope of $R$ vs $\partial B_{z} / \partial z$, for vertical (horizontal) gradients in $B_{z}$. Typically, $\partial B_{z} / \partial x$ may be $30-50 \mathrm{pT} / \mathrm{cm}$. in intermediate regimes, $\Delta h$ may be derived from a more precise expression for the center of mass $[1,15]$.

We now define an effective height difference

$$
\Delta h_{\mathrm{eff}}=\hat{z}_{\mathrm{Hg}}-\frac{\iint z \alpha(\epsilon)^{2} \rho(z, \epsilon) d z d \epsilon}{\iint \alpha(\epsilon)^{2} \rho(z, \epsilon) d z d \epsilon},
$$

which takes into account the relative contribution of each energy bin to the frequency measurement. The intrinsicdepolarization induced fractional decrease in the frequency ratio, away from that anticipated by Eq. (2), is then $\Delta(\delta R) / \delta R=\left(\Delta h-\Delta h_{\mathrm{eff}}\right) / \Delta h$. This function is shown (solid blue line) in Fig. 3. We see that the effect stays at the $2 \%$ level or below until quite large vertical gradients, in excess of $500 \mathrm{pT} / \mathrm{cm}$, by which time (as we shall see in Sec. VII below) the Ramsey wrapping will in any case long since have taken hold.

\section{B. Horizontal gradients: $\partial B_{z} / \partial x$}

We now carry out exactly the same calculations for the case of horizontal changes $\partial B_{z} / \partial x$ in the vertical magnetic field. The cylindrical trap is in this case to be bisected by a vertical rather than a horizontal plane, giving

$$
t_{w}=\frac{\pi r}{v} \text {. }
$$

This then yields

$$
T_{2, \mathrm{hgi}} \sim \frac{9 \pi v}{8 r^{3} \gamma_{n}^{2}\left(\partial B_{z} / \partial x\right)^{2}}
$$

where the subscript "hgi" indicates that this contribution arises from intrinsic depolarization due to the horizontal gradient.

Once again, this result (lower solid blue line in Fig. 2) provides a very reasonable approximation to our simulations (red triangles-upwards pointing, diffuse; downwards pointing, $80 \%$ specular). The $\sim 1.5$ orders of magnitude difference in response between the vertical and horizontal gradients arises principally because the bottle is 4 times wider than it is tall, and the respective dimensions enter to the third power. Figure 3 shows (green dot-dashed line) $\alpha$ as a function of the applied horizontal gradient.

Within our apparatus, it is difficult to tune the horizontal gradient in $B_{0}$ to better than about $30 \mathrm{pT} / \mathrm{cm}$, corresponding to $1 \mathrm{nT}$ (one part per thousand) difference from one side of the bottle to the other. We note that $50 \mathrm{pT} / \mathrm{cm}$ would yield a $T_{2}$ of $700 \mathrm{~s}$, perfectly consistent with that typically observed in the actual experiment and able to explain the reduction from an initial polarization of $\alpha=0.86$ when the trap is first filled to $\alpha=0.67$ at $220 \mathrm{~s}$ storage time in the absence of a vertical gradient. 
We can also calculate, just as we did for the vertical gradient, the fractional decrease in the frequency ratio $R$ that we might expect to see as a result of the spectral dependence of the intrinsic depolarization in this horizontal magnetic-field gradient. This is shown as a red dashed line in Fig. 3. We see that at horizontal gradients of around $50 \mathrm{pT} / \mathrm{cm}$, the slope of $R$ vs $\partial B_{z} / \partial z$ [Eq. (2)] decreases by about $10 \%$. This factor would be a constant, independent of the applied vertical gradient-it would not impose any curvature upon the vertical-gradient dependence. However, these calculations are for illustration only: we remind the reader that the frequency averaging implicit here is invalid when using the Ramsey resonance technique.

\section{Horizontal fields: $\partial B_{t} / \partial x$}

We consider here additional weak fields $B_{t}$ that are everywhere parallel to the $x y$ plane. If uniform, such fields simply act to produce a small tilt in the net direction of the main holding field $B_{0}$, and-since the perpendicular components add quadratically - a tiny change in its magnitude. The resulting field would still be uniform, leaving both the depolarization rate and the frequency ratio $R$ unaltered.

Such horizontal fields may of course have gradients of their own, e.g. if they are quadrupole-type fields of the form $B_{x}=q y, B_{y}=q x$, as discussed in Sec. VI.c of [4]. The direction of the total field will alter slightly from one side of the bottle to the other, but the UCN spins follow these changes adiabatically during their trajectory.

To understand the process in simple terms, let us first consider a neutron polarized with its spin along the $\hat{z}$ axis. If the cell has a difference $\Delta B_{t}$ in a transverse (i.e. horizontal; $x$ or $y$ ) field component from one side to the other, then on traversing the cell the UCN sees the $\vec{B}$ field tilt through an angle $\phi=\Delta B_{t} / B_{z}$ in a time $t_{c}=2 r / v_{t}$, where $v_{t}$ is the relevant transverse velocity. The angular frequency of this tilting motion is therefore $\omega_{\text {tilt }}=$ $\left(\Delta B_{t} / B_{z}\right) \cdot\left(v_{t} / 2 r\right)$. To keep $\vec{B}$ steady, we go to a reference frame rotating at $\omega_{f}=\omega_{\text {tilt }}$. To see the correct spin motion in this frame, we have to add the field

$$
B_{t}^{\prime}=\frac{1}{\gamma_{n}} \omega_{f}=\frac{\Delta B_{t}}{B_{z}} \frac{v_{t}}{2 \gamma_{n} r}
$$

A new $B_{t}^{\prime}$ must be used after each wall collision, since $\omega_{f}$ changes abruptly at that point. The result is that the spin of any one UCN executes a random walk, tracing out cones of small opening angles $\theta_{1}, \theta_{2}, \theta_{3}, \ldots$, where $\theta=B_{t}^{\prime} / B_{z}$, in the vicinity of the $\hat{z}$ direction. Assuming $N$ such wall collisions during a storage time $t=N t_{c}$, these small angles add vectorially to give a total angular displacement of magnitude

$$
\theta_{t}=\sqrt{N}\langle\theta\rangle=\sqrt{t} \frac{v_{t}}{2 r} \frac{B_{t}^{\prime}}{B_{z}} .
$$

Following the same methodology as for Eq. (9), and substituting for $B_{t}^{\prime}$ from Eq. (15), we arrive at

$$
T_{1, \mathrm{ffi}} \sim \frac{80 r^{3} \gamma_{n}^{2}}{v^{3}} \frac{B_{z}^{4}}{\Delta B_{t}^{2}},
$$

where we now refer to the longitudinal spin-relaxation time $T_{1}$ rather than $T_{2}$ because we began with the spin aligned along $\hat{z}$. The subscript "tfi" refers to "transverse-field, intrinsic," and we have taken $v_{t}^{2}=v^{2} / 3$. This derivation is of course extremely simplistic (for example, use of the mean free path $\lambda$ rather than the cell diameter would immediately reduce $T_{1, \text { ffi }}$ by a factor $\sim 3$ for our trap geometry). However, it gives interesting insight, and (given that we are in the "high-field" regime where the spinprecession frequency is substantially higher than the collision frequency) we note that its dependence upon parameters is identical to that of the rather more sophisticated Eq. (66) in [28].

This particular depolarization mechanism is less effective by a factor of 2 when acting upon a spin precessing in the horizontal plane rather than aligned with $\hat{z}$, for the simple reason that $B_{x}$ components do not affect the $x$ component of spin, and similarly for $y$ components. This leads to the well-known result for this case [23]:

$$
T_{2, \mathrm{tfi}}=2 T_{1, \mathrm{tfi}} \text {. }
$$

Putting in realistic numbers for our apparatus (a few nT for $\Delta B_{t}$, and $v \sim 2 \mathrm{~m} / \mathrm{s}$ ), we find that Eq. (17) predicts $T_{1, \mathrm{tfi}}$ (and therefore $T_{2, \mathrm{ffi}}$ ) values of order $10^{6}$ seconds. We certainly cannot expect to be sensitive to this. In any case, we have observed $T_{1}$ times in excess of $1000 \mathrm{~s}$, implying that $T_{2, \mathrm{ffi}}>2000 \mathrm{~s}$, so this is clearly not a dominant effect.

In terms of frequency shifts, the mercury atoms will average out the horizontal components, whereas the neutrons remain sensitive to the total field magnitude. This gives rise to a change in the frequency ratio $R$ of

$$
\frac{\delta R}{R}=\frac{q^{2} r^{2}}{4 B_{0}^{2}}
$$

where, as before, the radius of the trap is $r$. This will be a constant shift, independent of the applied vertical gradient. In the case of the EDM spectrometer, where horizontal field components are several hundred to a thousand times smaller than the vertical field, the resulting frequency shifts are of the order of a part per million or less.

\section{Wall collisions}

The cell walls may contain tiny magnetic impurities. Collisions with these would disturb the spins on time scales 
much shorter than the Larmor precession period. Since such perturbations can affect any orientation of spin equally, one can anticipate that

$$
T_{2, \mathrm{wall}}=T_{1, \mathrm{wall}} \text {. }
$$

As noted above, we have measured $T_{1}$ to be in excess of $1000 \mathrm{~s}$, which therefore sets a lower limit of $1000 \mathrm{~s}$ on the contribution to $T_{2}$ arising from wall collisions. This is therefore unlikely to be a significant source of depolarization.

\section{POLARIZATION VS APPLIED VERTICAL GRADIENT}

Having established the expected response to the intrinsic depolarization, we now go on to look at the effects of the gravitationally enhanced depolarization, using calculations as discussed in Sec. III above.

We show in Fig. 4 the residual polarization, after $180 \mathrm{~s}$ of storage, as a function of the applied vertical magnetic-field gradient. The data points (black triangles) represent measurements [9] made with the magnetic holding field $B_{0}$ pointing downwards. These measurements were made in 2012, more than two years before the spin-echo measurements of the UCN spectrum, but we have no reason to suspect that the spectrum would have changed during the intervening period. The solid line in light magenta shows the approximate expected contribution of the intrinsic depolarization, based on the formulas of Eqs. (9) and (14); its profile should be correct, although there is uncertainty over its scale because we do not know the

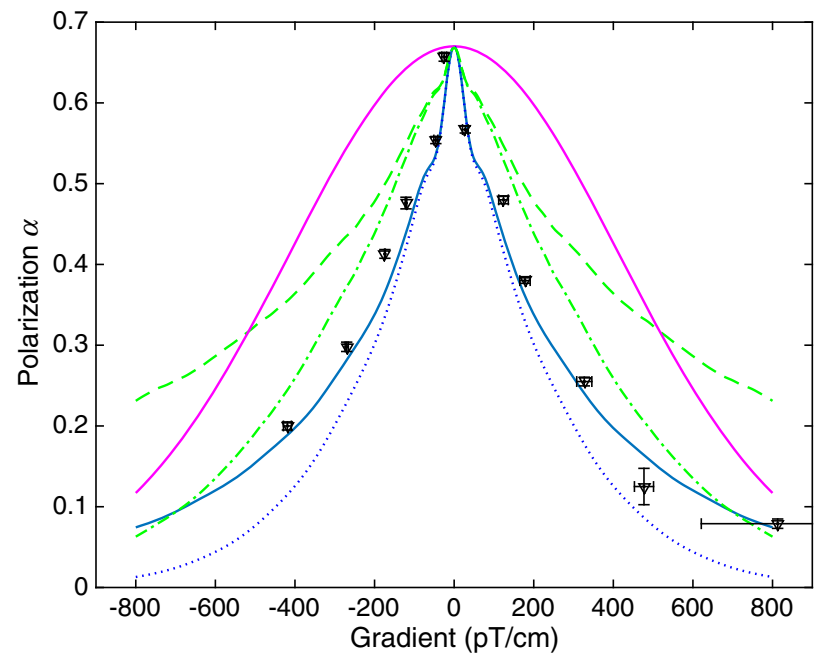

FIG. 4 (color online). Depolarization as a function of applied vertical magnetic-field gradient. Light magenta line: calculated intrinsic contribution. Blue solid and green dashed lines are the enhanced contributions applied to the SE and MC spectra, respectively. The blue dotted and green dot-dashed lines show the combined effects. Measured data points are represented as black triangles. extent to which reflection of UCN within the trap is specular. The blue solid (green dashed) line shows the contribution of gravitationally enhanced depolarization, using the measured SE (MC) spectrum as input. The blue dotted (green dot-dashed) line shows the combined calculated effect. In each case the calculated profiles are normalized to reflect the peak measured value of 0.67 , which is a result both of imperfect initial polarization and also of intrinsic depolarization e.g. from horizontal field gradients of the form $\partial B_{z} / \partial x . B_{0}$ up data are omitted from this plot for clarity; they are similar in form to the data shown, but with a somewhat lower maximum value of about $55 \%$.

There are two striking features about this plot. The first is the very distinctive peaked shape of the profile near the maximum. The intrinsic depolarization mechanism has a very soft peak, parabolic in nature. In contrast, the gravitationally enhanced component is almost triangular in form, precisely mirroring the behavior of the data.

The second feature of interest is the close match in the polarization profile across a wide range of gradients. No parameters were optimized in the calculated curves beyond the normalization of the peak value (equivalent to assuming $\left.\partial B_{z} / \partial x=50 \mathrm{pT} / \mathrm{cm}\right)$. As noted above, the MC spectrum is expected to be a little too hard, and we see that the data lie below the corresponding (green) lines as one would anticipate. The measured SE spectrum, on the other hand, is known to be a little too soft, since it is representative of a 220 s storage time whereas the data points were measured at $180 \mathrm{~s}$. One would therefore expect the combined effects of intrinsic and enhanced contributions (dotted blue) to lie a little below the data points, as indeed they do. It would appear that, rather fortuitously, the offset from the use of a softened spectrum is here almost exactly compensated by the additional contribution of the intrinsic depolarization, leaving the calculated enhanced contribution more or less perfectly aligned with the data.

\section{FREQUENCY SHIFTS AT LARGE VERTICAL FIELD GRADIENTS}

We now turn to measurements of the ratio of neutron to mercury precession frequencies under applied vertical magnetic-field gradients.

Figure 5 shows the measured data alongside the results from calculations of the effect of gravitationally enhanced depolarization, based upon the measured SE (solid blue line) and simulated MC (dashed green line) spectra discussed in Sec. II above. The adjacent blue dotted and green dot-dashed lines include the approximate respective contributions from intrinsic depolarization, with the sines and cosines of the contributing phases [see Eq. (5)] weighted as $\alpha^{2}$. As anticipated, the intrinsic depolarization has little additional effect.

Both the SE and the MC spectra result in the right general trend, i.e. curvature of the appropriate form. 
S. AFACH et al.

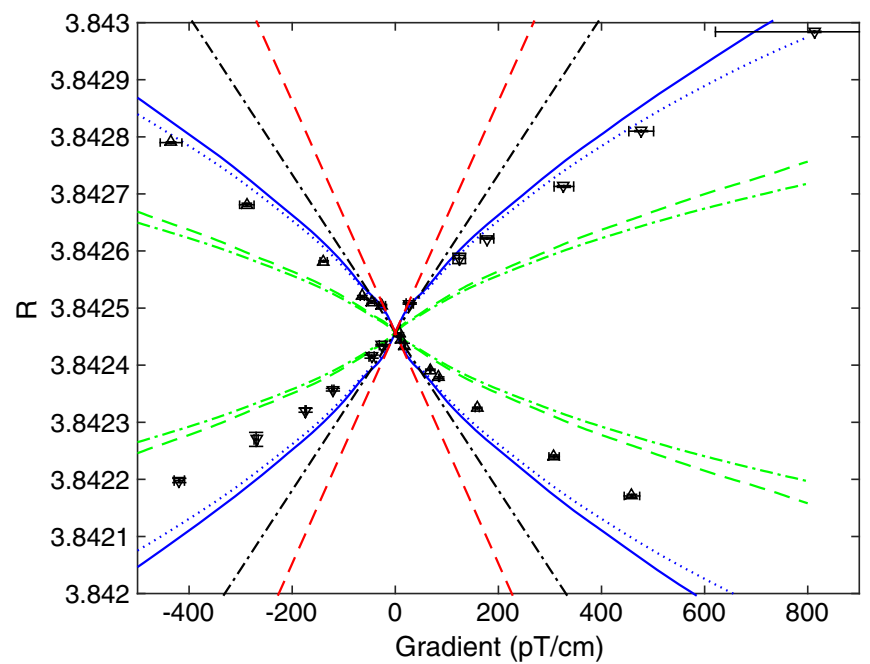

FIG. 5 (color online). Ratio of neutron to mercury frequencies as a function of the applied vertical magnetic-field gradient. Triangular upwards-pointing data points (running downwards diagonally) are for $B_{0}$ up; triangular downwards-pointing data points (running upwards diagonally) are for $B_{0}$ down. Red dashed lines show the expectation from Eq. (2), using $\Delta h$ from the SE spectrum. The black dot-dashed lines represent a fit to data in the central region, with gradients of less than $60 \mathrm{pT} / \mathrm{cm}$. The blue solid (green dashed) line includes gravitationally enhanced depolarization, based on the SE (MC) spectrum, with the adjacent blue dotted (green dot-dashed) lines including the effect of intrinsic depolarization.

However, as one might expect, the (stiffer) simulated spectrum results in curves that are less steep than the data, whereas the (softer) spin-echo spectrum, with its larger $\Delta h$, results in curves that are rather steeper.

Also shown in this figure as a pair of red dashed lines is the expected response based on Eq. (2), using the $\Delta h$ from the SE spectrum (with no depolarization), as well as (black dot-dashed lines) a fit to the data points with vertical gradients of less than $60 \mathrm{pT} / \mathrm{cm}$. Bearing in mind both that the SE spectrum is a little too soft and that no depolarization effects at all are included, one would anticipate that the former lines would be slightly steeper than the latter, as is indeed the case.

\section{FREQUENCY SHIFTS AT SMALL VERTICAL FIELD GRADIENTS}

The effect that we wish to discuss here is arguably more subtle. We focus upon the very central region of the frequency-ratio curves, as shown in Fig. 6. We again show (via the blue solid and green dashed lines, respectively) the results of calculations of gravitationally enhanced depolarization based upon the SE and MC spectra.

It is apparent that there is a change in the slope of the lines as the crossing point is approached. It is visible in the data, where the trend at higher gradients is highlighted by the black dot-dashed lines: these represent a common fit of
PHYSICAL REVIEW D 92, 052008 (2015)

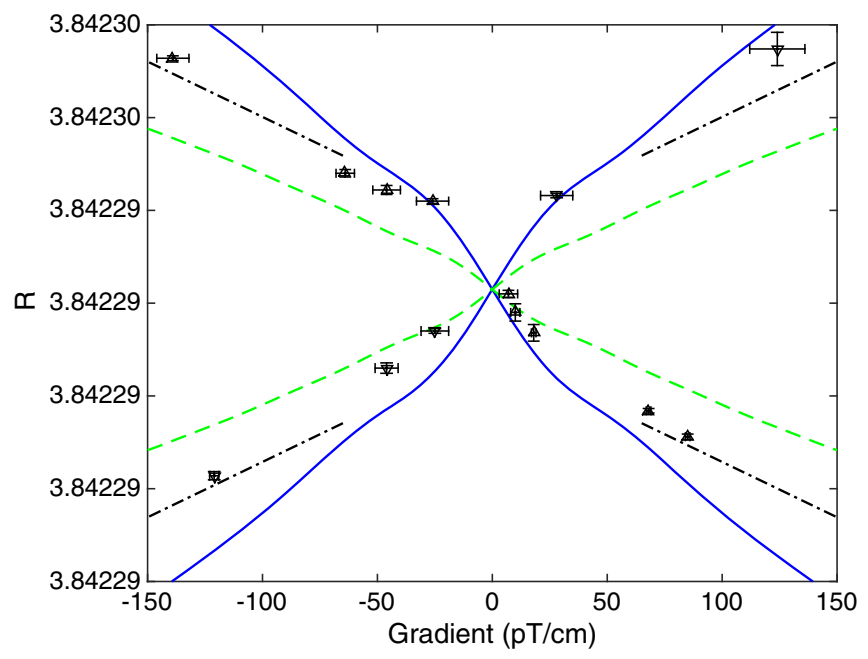

FIG. 6 (color online). A closeup of the central region of the curves showing the dependence of the ratio of neutron to mercury frequencies upon the applied vertical magnetic-field gradient. Triangular upwards- and downwards-pointing data markers once again represent measurements taken with the holding field $B_{0}$ aligned vertically upwards and downwards respectively. Solid blue and dashed green lines again arise from calculations based on the spin-echo and simulated spectra, respectively. The black dot-dashed lines in the corner regions represent the trend of the data at higher gradients.

all of the data with gradients of more than $100 \mathrm{pT} / \mathrm{cm}$ to the function

$$
\left|R-R_{0}\right|=m\left|\frac{\partial B_{z}}{\partial z}\right|+c,
$$

where $R_{0}=3.8424574$ is the crossing-point value of $R$ [9]. If extrapolated to lower gradients, these lines would clearly result in a significant discontinuity.

We ascribe this phenomenon to Ramsey wrapping, which makes the spreading low-energy tail of the array of integrated phases indistinguishable from the contributions of high-energy UCN. This moderates the frequency shift as described in [1] and shown in Fig. 4 therein. Intuitively, we would expect this to start happening when the difference in magnetic field between the bottom of the bottle (where the low-energy UCN preferentially spend their time) and the center of the bottle (which is the average position for higher-energy neutrons) is sufficient to produce a phase shift of between $\pi$ and $2 \pi$ over the $180 \mathrm{~s}$ storage time. This amounts to about 100-200 pT over the $6 \mathrm{~cm}$ halfheight, i.e. a gradient of about $15-30 \mathrm{pT} / \mathrm{cm}$, which is precisely where we observe it happening.

The particularly keen-eyed reader may be able to perceive a further slight curvature in the calculated curve at a gradient of about $\pm 50 \mathrm{pT} / \mathrm{cm}$. This is due to that same low-energy tail wrapping itself around for a second time. 
The data do not have adequate resolution to discern this effect.

We note finally that both the curves and the real physical behavior of the system are expected to be symmetric with respect to the crossing point (indeed, the calculations represented here were generated in a single quadrant only, and then reflected through $\partial B_{z} / \partial z=0$ and through $R=R_{0}$ ). Therefore, data taken at points using the same positive and negative gradients and with both $B_{0}$ field directions would allow one to extract the correct crossing point without knowing the curvature. This is an important safeguard for future nEDM data taking.

\section{IMPLICATIONS FOR nEDM}

The leading systematic error in the nEDM measurement described in [5] arose from shifts in the Larmor precession frequency brought about by the interplay between (a) small magnetic-field gradients within the apparatus and (b) the motional magnetic fields due to the particles (both UCN and, in particular, the mercury atoms used for magnetometry) moving through the applied electric field [4]. This effect was compensated by considering the behavior of the observed apparent EDM signal over a range of magneticfield gradients. There was no direct measurement of the field gradient: instead, it was parametrized [Eq. (2)] by the ratio $R$ of neutron to mercury precession frequencies. It is now clear, however, that even at quite moderate gradients $R$ is subject to the nonlinearities discussed above. Furthermore, it is stated in [5] that the height difference $\Delta h$ between the UCN and the mercury atoms was $2.8 \mathrm{~mm}$, with a precision of $4 \%$. This latter was based upon measurements of the frequency response within a variable-height trap; in fact those measurements also would have been affected by gravitationally enhanced depolarization. The UCN energy spectrum was undoubtedly softer than had been thought, implying that the calculated slope of the lines in Fig. 2 of [5] was steeper than it should have been. The relatively good match of the calculated to the actual slope of the fitted line is due in part to the nonlinear nature of the dependence of $R$ upon $\partial B_{z} / \partial z$, which would be similar in form to that shown in Fig. 6 above and in Fig. 4 of [1], and which would result in a steeper-than-expected slope once beyond the linear region.

Since the data were taken more or less symmetrically about the crossing point, this effect is unlikely to produce a very substantial change in the nEDM limit in this case. Nonetheless, a detailed reanalysis is now underway, and it is expected that a revised result will emerge shortly. Future measurements will doubtless enjoy the advantage of better diagnostics both of the magnetic field (with improved magnetometry) and of the energy spectrum (using the spinecho technique).

\section{CONCLUSIONS}

Measurements undertaken at the EDM spectrometer at PSI, showing the dependence upon applied vertical magnetic-field gradients of depolarization rates and of the neutron precession-frequency, have clearly demonstrated features that are characteristic of the anticipated behavior resulting from gravitationally enhanced depolarization and Ramsey wrapping: namely, a sharply peaked rather than parabolic depolarization profile, and significant nonlinearities in the frequency-response curve. Using estimates of the spectrum of stored UCN, based upon measurements using the spin-echo technique and also upon detailed simulations, we have demonstrated excellent qualitative agreement between measurements and theoretical expectations. It also seems clear that intrinsic depolarization processes have only a marginal effect upon frequency shifts in the presence of magnetic-field gradients, and that such shifts are dominated by the gravitationally enhanced component.

There are obvious implications for nEDM measurements, including for the analysis that led to the current world limit [5], since the frequency-response curve is used to correct for systematic effects.

\section{ACKNOWLEDGMENTS}

We would like to thank the PSI staff, in particular F. Burri and M. Meier, for their outstanding support. We also gratefully acknowledge the important work carried out by the mechanical workshop of the University of Fribourg Physics Department. This research was financed in part by the Fund for Scientific Research, Flanders; Grant No. GO A/2010/10 of KU Leuven; the Swiss National Science Foundation Projects No. 200020-144473 (PSI), No. 200021-126562 (PSI), No. 200020-149211 (ETH) and No. 200020-140421 (Fribourg); and Grants No. ST/ K001329/1, No. ST/M003426/1 and No. ST/L006472/1 from the UK's Science and Technology Facilities Council (STFC). One of us (E. W.) is a Ph.D. Fellow of the Research Foundation-Flanders (FWO). The original apparatus was funded by grants from the UK's PPARC (now STFC). The LPC Caen and the LPSC acknowledge the support of the French Agence Nationale de la Recherche (ANR) under Reference No. ANR-09-BLAN-0046. Polish partners wish to acknowledge support from the PL-Grid infrastructure and from the National Science Centre, Poland, under Grant No. UMO-2012/04/M/ST2/00556. 
[1] P. G. Harris, J. M. Pendlebury, and N. E. Devenish, Gravitationally enhanced depolarization of ultracold neutrons in magnetic-field gradients, Phys. Rev. D 89, 016011 (2014).

[2] A. Knecht, Ph.D. thesis, Universität Zürich, 2009.

[3] B. Franke, Ph.D. thesis, Universität Zürich, 2013.

[4] J. M. Pendlebury, W. Heil, Y. Sobolev, P. G. Harris, J. D. Richardson, R. J. Baskin, D. D. Doyle, P. Geltenbort, K. Green, M. G. D. van der Grinten et al., Geometric-phaseinduced false electric dipole moment signals for particles in traps, Phys. Rev. A 70, 032102 (2004).

[5] C. A. Baker, D. D. Doyle, P. Geltenbort, K. Green, M. G. D. van der Grinten, P. G. Harris, P. Iaydjiev, S. N. Ivanov, D. J. R. May, J. M. Pendlebury et al., Improved Experimental Limit on the Electric Dipole Moment of the Neutron, Phys. Rev. Lett. 97, 131801 (2006).

[6] R. Golub and S. K. Lamoreaux, Neutron electric-dipole moment, ultracold neutrons and polarized ${ }^{3} \mathrm{He}$, Phys. Rep. 237, 1 (1994).

[7] C. Baker, G. Ban, K. Bodek, M. Burghoff, Z. Chowdhuri, M. Daum, M. Fertl, B. Franke, P. Geltenbort, K. Green et al., The search for the neutron electric dipole moment at the Paul Scherrer Institute, Phys. Procedia 17, 159 (2011).

[8] B. Lauss, Ultracold neutron production at the second spallation target of the Paul Scherrer Institute, Phys. Procedia 51, 98 (2014).

[9] S. Afach, C. Baker, G. Ban, G. Bison, K. Bodek, M. Burghoff, Z. Chowdhuri, M. Daum, M. Fertl, B. Franke et al., A measurement of the neutron to ${ }^{199} \mathrm{Hg}$ magnetic moment ratio, Phys. Lett. B 739, 128 (2014).

[10] C. Baker, Y. Chibane, M. Chouder, P. Geltenbort, K. Green, P. Harris, B. Heckel, P. Iaydjiev, S. Ivanov, I. Kilvington et al., Apparatus for measurement of the electric dipole moment of the neutron using a cohabiting atomic-mercury magnetometer, Nucl. Instrum. Methods Phys. Res., Sect. A 736, 184 (2014).

[11] P. Knowles, G. Bison, N. Castagna, A. Hofer, A. Mtchedlishvili, A. Pazgalev, and A. Weis, Laser-driven Cs magnetometer arrays for magnetic field measurement and control, Nucl. Instrum. Methods Phys. Res., Sect. A 611, 306 (2009).

[12] S. Afach et al., A device for simultaneous spin analysis of ultracold neutrons, arXiv:1502.06876 (to be published).

[13] S. Afach, G. Bison, K. Bodek, F. Burri, Z. Chowdhuri, M. Daum, M. Fertl, B. Franke, Z. Grujic, V. Hélaine et al., Dynamic stabilization of the magnetic field surrounding the neutron electric dipole moment spectrometer at the Paul Scherrer Institute, J. Appl. Phys. 116, 084510 (2014).

[14] K. Green, P. Harris, P. Iaydjiev, D. May, J. Pendlebury, K. Smith, M. van der Grinten, P. Geltenbort, and S. Ivanov, Performance of an atomic mercury magnetometer in the neutron EDM experiment, Nucl. Instrum. Methods Phys. Res., Sect. A 404, 381 (1998).

[15] S. Afach, N. Ayres, G. Ban et al., Observation of Gravitationally Induced Vertical Striation of Polarized Ultracold Neutrons by Spin-Echo Spectroscopy, arXiv:1506.00446 [Phys. Rev. Lett. (to be published)].

[16] K. Bodek, Z. Chowdhuri, M. Daum, M. Fertl, B. Franke, E. Gutsmiedl, R. Henneck, M. Horras, M. Kasprzak, K. Kirch et al., MC calculations for the nEDM experiment systematics, Phys. Procedia 17, 259 (2011).

[17] R. Golub, D. Richardson, and S. Lamoreaux, Ultra-Cold Neutrons (Adam Hilger, Bristol, 1991).

[18] M. Kuźniak, Ph.D. thesis, Jagiellonian University, 2008.

[19] J. Pendlebury and D. Richardson, Effects of gravity on the storage of ultracold neutrons, Nucl. Instrum. Methods Phys. Res., Sect. A 337, 504 (1994).

[20] L. D. Schearer and G. K. Walters, Nuclear spin-lattice relaxation in the presence of magnetic-field gradients, Phys. Rev. 139, A1398 (1965).

[21] G. D. Cates, S. R. Schaefer, and W. Happer, Relaxation of spins due to field inhomogeneities in gaseous samples at low magnetic fields and low pressures, Phys. Rev. A 37, 2877 (1988).

[22] G. D. Cates, D. J. White, T.-R. Chien, S. R. Schaefer, and W. Happer, Spin relaxation in gases due to inhomogeneous static and oscillating magnetic fields, Phys. Rev. A 38, 5092 (1988).

[23] D. D. McGregor, Transverse relaxation of spin-polarized ${ }^{3} \mathrm{He}$ gas due to a magnetic field gradient, Phys. Rev. A 41, 2631 (1990).

[24] R. Schmid, B. Plaster, and B. W. Filippone, Motional spin relaxation in large electric fields, Phys. Rev. A 78, 023401 (2008).

[25] S. K. Lamoreaux and R. Golub, Detailed discussion of a linear electric field frequency shift induced in confined gases by a magnetic field gradient: Implications for neutron electric-dipole-moment experiments, Phys. Rev. A 71, 032104 (2005).

[26] R. Golub, R. M. Rohm, and C. M. Swank, Reexamination of relaxation of spins due to a magnetic field gradient: Identity of the Redfield and Torrey theories, Phys. Rev. A 83, 023402 (2011).

[27] G. Pignol and S. Roccia, Electric-dipole-moment searches: Reexamination of frequency shifts for particles in traps, Phys. Rev. A 85, 042105 (2012).

[28] D. Kleppner, H. M. Goldenberg, and N. F. Ramsey, Theory of the hydrogen maser, Phys. Rev. 126, 603 (1962).

[29] J. Pendlebury, Kinetic Theory, Student Monographs in Physics (Adam Hilger, Bristol, 1985). 